\title{
OSCILLATION OF HIGHER-ORDER DELAY DIFFERENCE EQUATIONS
}

YINGGAO ZHOU

Received 6 January 2006; Revised 18 April 2006; Accepted 20 April 2006

The oscillation and asymptotic behavior of the higher-order delay difference equation $\Delta^{l} x_{n}+\sum_{i=1}^{m} p_{i}(n) x_{n-k_{i}}=0, n=0,1,2, \ldots$, are investigated. Some sufficient conditions of oscillation and bounded oscillation of the above equation are obtained.

Copyright (c) 2006 Yinggao Zhou. This is an open access article distributed under the Creative Commons Attribution License, which permits unrestricted use, distribution, and reproduction in any medium, provided the original work is properly cited.

\section{Introduction}

Consider the following delay difference equation:

$$
\triangle^{l} x_{n}+\sum_{i=1}^{m} p_{i}(n) x_{n-k_{i}}=0, \quad n=0,1,2, \ldots,
$$

and its first-order corresponding inequality

$$
\triangle x_{n}+\sum_{i=1}^{m} p_{i}(n) x_{n-k_{i}} \leq 0, \quad n=0,1,2, \ldots,
$$

where $\left\{p_{i}(n)\right\}$ are sequences of nonnegative real numbers and not identically equal to zero, and $k_{i}$ is positive integer, $i=1,2, \ldots$, and $\triangle$ is the first-order forward difference operator, $\triangle x_{n}=x_{n+1}-x_{n}$, and $\triangle^{l} x_{n}=\triangle^{l-1}\left(\triangle x_{n}\right)$ for $l \geq 2$.

By a solution of (1.1) or inequality (1.2), we mean a nontrival real sequence $\left\{x_{n}\right\}$ satisfying (1.1) or inequality (1.2) for $n \geq 0$. A solution $\left\{x_{n}\right\}$ is said to be oscillatory if it is neither eventually positive nor eventually negative and nonoscillatory otherwise. An equation is said to be oscillatory if its every solution is oscillatory.

The oscillatory behavior of difference equations has been intensively studied in recent years. Most of the literature has been concerned with equations of type (1.1) with $l=1$ (see [1-10] and references cited therein). But very little is known regarding the oscillation of higher-order difference equation similar to (1.1). The purpose of this paper is to study the oscillatory properties of (1.1).

Hindawi Publishing Corporation

Advances in Difference Equations

Volume 2006, Article ID 65789, Pages 1-7

DOI 10.1155/ADE/2006/65789 
2 Oscillation of higher-order delay difference equations

\section{Main results}

We need the following several lemmas in order to prove our results.

Lemma $2.1[5,8]$. Assume that

$$
\liminf _{n \rightarrow \infty} \sum_{i=1}^{m}\left(\frac{k_{i}+1}{k_{i}}\right)^{k_{i}+1} \sum_{s=n+1}^{n+k_{i}} p_{i}(s)>1,
$$

or

$$
\limsup _{n \rightarrow \infty} \sum_{i=1}^{m} \sum_{s=n}^{n+k_{i}} p_{i}(s)>1 .
$$

Then inequality (1.2) has no eventually positive solution.

Lemma 2.2 [1]. Let $x_{n}$ be defined for $n \geq n_{0}$ and $x_{n}>0$ with $\triangle^{l} x_{n}$ eventually of one sign and not identically zero. Then there exist an integer $j, 0 \leq j \leq l$ with $(l+j)$ odd for $\triangle^{l} x_{n} \leq 0$ and $(l+j)$ even for $\triangle^{l} x_{n} \geq 0$ and an integer $N \geq n_{0}$, such that for all $n \geq N$,

$$
\begin{gathered}
j \leq l-1 \Longrightarrow(-1)^{j+i} \triangle^{i} x_{n}>0, \quad j \leq i \leq l-1, \\
j \geq 1 \Longrightarrow \triangle^{i} x_{n}>0, \quad 1 \leq i \leq j-1 .
\end{gathered}
$$

Specially, if $\triangle^{l} x_{n} \leq 0$ for $n \geq n_{0}$, and $\left\{x_{n}\right\}$ is bounded, then

$$
\begin{gathered}
(-1)^{i+1} \triangle^{l-i} x_{n} \geq 0, \quad \forall \text { large } n \geq n_{0}, i=1, \ldots, l-1, \\
\lim _{n \rightarrow \infty} \triangle^{i} x_{n}=0, \quad 1 \leq i \leq l-1 .
\end{gathered}
$$

Lemma 2.3 [1]. Let $x_{n}$ be defined for $n \geq n_{0}$, and $x_{n}>0$ with $\triangle^{l} x_{n} \leq 0$ for $n \geq n_{0}$ and not identically zero. If $x_{n}$ is increasing, then there exists a large integer $n_{1} \geq n_{0}$ such that

$$
x_{n} \geq \frac{2^{2-2 l}}{(l-1) !} n^{(l-1)} \triangle^{l-1} x_{n}, \quad \forall n \geq 2^{l} n_{1} .
$$

Specially,

$$
x_{n} \geq \frac{\theta}{(l-1) !} n^{l-1} \triangle^{l-1} x_{n}, \quad \text { for sufficiently large } n,
$$

where $0<\theta<1$ with $\lim _{n \rightarrow \infty} \theta=1$, and $n^{(t)}=n(n-1) \cdots(n-t+1)$, for every nonnegative integer $t$, and $n^{(0)}=1$.

Theorem 2.4. Assume that

$$
\liminf _{n \rightarrow \infty} \sum_{i=1}^{m}\left(\frac{k_{i}+1}{k_{i}}\right)^{k_{i}+1} \sum_{s=n+1}^{n+k_{i}} p_{i}(s)>(l-1) ! .
$$

Then every solution $x_{n}$ of (1.1) oscillates, or $x_{n} \rightarrow 0(n \rightarrow \infty)$. 
Proof. Assume, for the sake of contradiction, that $\left\{x_{n}\right\}$ is an eventually positive solution of (1.1), then there exists a positive integer $N_{1}$ such that

$$
x_{n}>0, \quad x_{n-k_{i}}>0, \quad i=1, \ldots, m, n \geq N_{1} \text {. }
$$

Thus,

$$
\triangle^{l} x_{n}=-\sum_{i=1}^{m} p_{i}(n) x_{n-k_{i}} \leq 0, \quad n \geq N_{1}
$$

and $\triangle^{l} x_{n} \not \equiv 0$.

By Lemma 2.2, $\triangle^{i} x_{n}$ are eventually of one sign for every $i \in\{1, \ldots, l-1\}$ and $\triangle^{l-1} x_{n}>0$ holds for large $n$, and there exist two cases to consider: (1) $\triangle x_{n}>0$ and (2) $\triangle x_{n}<0$.

Case 1. This says that $x_{n}$ is increasing. Setting $k=\max \left\{k_{1}, \ldots, k_{m}\right\}$, by Lemma 2.3, there exists an integer $N_{2} \geq \max \left\{k, N_{1}\right\}$ such that

$$
\begin{gathered}
x_{n} \geq \frac{\theta}{(l-1) !} n^{l-1} \triangle^{l-1} x_{n}, \quad n \geq N_{2}, \\
x_{n-k_{i}} \geq \frac{\theta}{(l-1) !}\left(n-k_{i}\right)^{l-1} \triangle^{l-1} x_{n-k_{i}} \\
\geq \frac{\theta}{(l-1) !}(n-k)^{l-1} \triangle^{l-1} x_{n-k_{i}}, \quad i=1, \ldots, m, n \geq N_{2},
\end{gathered}
$$

where $0<\theta<1$ and $\lim _{n \rightarrow \infty} \theta=1$.

Letting $y_{n}=\triangle^{l-1} x_{n}$, we have

$$
y_{n}>0, \quad y_{n-k_{i}}>0, \quad i=1, \ldots, m, n \geq N_{2},
$$

which implies that

$$
\triangle y_{n}+\sum_{i=1}^{m} p_{i}(n) x_{n-k_{i}}=0, \quad n \geq N_{2} .
$$

By (2.11), we get

$$
\begin{gathered}
x_{n-k_{i}} \geq \frac{\theta}{(l-1) !}(n-k)^{l-1} y_{n-k_{i}}, \quad i=1, \ldots, m, n \geq N_{2}, \\
\geq \frac{\theta}{(l-1) !} y_{n-k_{i}}, \quad i=1, \ldots, m, n \geq N_{2} .
\end{gathered}
$$

It follows that

$$
\triangle y_{n}+\sum_{i=1}^{m} \tilde{p}_{i}(n) y_{n-k_{i}} \leq 0, \quad n \geq N_{2},
$$

where $\tilde{p}_{i}(n)=(\theta /(l-1) !) p_{i}(n)$, which means that inequality $(2.15)$ has an eventually positive solution. 
4 Oscillation of higher-order delay difference equations

On the other hand, condition (2.7) implies that

$$
\begin{aligned}
\liminf _{n \rightarrow \infty} & \sum_{i=1}^{m}\left(\frac{k_{i}+1}{k_{i}}\right)^{k_{i}+1} \sum_{s=n+1}^{n+k_{i}} \tilde{p}_{i}(s) \\
& =\liminf _{n \rightarrow \infty} \frac{\theta}{(l-1) !} \sum_{i=1}^{m}\left(\frac{k_{i}+1}{k_{i}}\right)^{k_{i}+1} \sum_{s=n+1}^{n+k_{i}} p_{i}(s)>1 .
\end{aligned}
$$

By Lemma 2.1, (2.15) has no eventually positive solution. This is a contradiction.

Case 2. Note that by Lemma 2.2, the case that $l$ is even is impossible. In what follows, we only consider the case that $l$ is odd. Case 2 says that $x_{n}$ is monotone and bounded, and so $x_{n}$ converges a constant $a$. By Lemma 2.2, we get

$$
\begin{gathered}
(-1)^{i+1} \triangle^{l-i} x_{n}>0, \quad i=1, \ldots, l-1, \quad \forall \text { large } n \geq N_{1}, \\
\lim _{n \rightarrow \infty} \triangle^{l-1} x_{n}=0 .
\end{gathered}
$$

By (2.18), there exists an integer $N_{3} \geq N_{1}$ such that

$$
0 \leq \triangle^{l-1} x_{n} \leq \varepsilon, \quad \text { for any } \varepsilon>0, n \geq N_{3}
$$

It is obvious that $a \geq 0$. If $a=0$, then the problem is solved. We can assume that $a>0$ in the sequel, which implies that there exists an integer $N_{4} \geq N_{3}$ such that

$$
x_{n}>\frac{1}{2} a, \quad x_{n-k_{i}}>\frac{1}{2} a, \quad i=1,2, \ldots, m, n \geq N_{4} .
$$

Thus, (1.1) implies that

$$
\triangle^{l} x_{n}+\frac{a}{2} \sum_{i=1}^{m} p_{i}(n) \leq 0, \quad n \geq N_{4}
$$

Summing both sides of (2.21) from $N_{4}$ to $n$, we obtain

$$
\triangle^{l-1} x_{n+1}-\triangle^{l-1} x_{N_{4}}+\frac{a}{2} \sum_{s=N_{4}}^{n} \sum_{i=1}^{m} p_{i}(s) \leq 0, \quad n \geq N_{4} .
$$

Letting $n \rightarrow \infty$, we have

$$
\frac{a}{2} \sum_{i=1}^{m} \sum_{s=N_{4}}^{n} p_{i}(s) \leq \varepsilon, \quad \text { for large } n .
$$

On the other hand, condition (2.7) says that there exists an integer $N_{5} \geq N_{4}$ such that

$$
\sum_{i=1}^{m}\left(\frac{k_{i}+1}{k_{i}}\right)^{k_{i}+1} \sum_{s=n+1}^{n+k_{i}} p_{i}(s)>\frac{(l-1) !}{2}, \quad n \geq N_{5} .
$$


Noting that $\left(\left(k_{i}+1\right) / k_{i}\right)^{k_{i}+1} \leq 2 e$, we have

$$
\frac{a}{2} \sum_{i=1}^{m} \sum_{s=n+1}^{n+k_{i}} p_{i}(s)>\frac{a(l-1) !}{8 e}, \text { for large } n,
$$

which contradicts (2.23) and (2.25). The proof is completed.

Similar to the proof of Theorem 2.4, we have Theorem 2.5.

Theorem 2.5. Assume that

$$
\limsup _{n \rightarrow \infty} \sum_{i=1}^{m} \sum_{s=n}^{n+k_{i}} p_{i}(s)>(l-1) !
$$

Then every solution $x_{n}$ of $(1.1)$ is oscillatory, or $x_{n} \rightarrow 0(n \rightarrow \infty)$.

In fact, in the proof of Theorem 2.4, the condition (2.26) implies that (2.25) always holds and (2.16) is changed into the following inequality:

$$
\limsup _{n \rightarrow \infty} \sum_{i=1}^{m} \sum_{s=n}^{n+k_{i}} \tilde{p}_{i}(s)>1 .
$$

The rest of proof is the same as the proof of Theorem 2.4.

Theorem 2.6. Assume that $l$ is even, and the following condition holds:

$$
\liminf _{n \rightarrow \infty} \sum_{i=1}^{m}\left(\frac{k_{i}+1}{k_{i}}\right)^{k_{i}+1} \sum_{s=n+1}^{n+k_{i}} s^{l-1} p_{i}(s)>(l-1) !
$$

Then every bounded solution $x_{n}$ of (1.1) oscillates.

Proof. Assume, for the sake of contradiction, that $x_{n}$ is an eventually positive bounded solution of (1.1). According to the proof of Theorem 2.4, there exists a positive integer $N_{1}$ such that (2.8) and (2.9) hold. By Lemma 2.2, we have

$$
\triangle x_{n}>0
$$

which implies that $x_{n}$ is increasing. In view of the proof of Theorem 2.4, there exists an integer $N_{2} \geq N_{1}$ such that

$$
x_{n-k_{i}} \geq \frac{\theta}{(l-1) !}(n-k)^{l-1} y_{n-k_{i}}, \quad i=1, \ldots, m, n>N_{2},
$$

where $k=\max \left\{k_{1}, \ldots, k_{m}\right\}, 0<\theta<1$ with $\lim _{n \rightarrow \infty} \theta=1$. It follows that

$$
\triangle y_{n}+\sum_{i=1}^{m} \tilde{p}_{i}(n) y_{n-k_{i}} \leq 0, \quad n \geq N_{2}
$$

where $\tilde{p}_{i}(n)=(\theta /(l-1) !)(n-k)^{l-1} p_{i}(n), y_{n}=\triangle^{l-1} x_{n}$, which implies that (2.31) has an eventually positive solution. 
6 Oscillation of higher-order delay difference equations

On the other hand, condition (2.28) implies that

$$
\begin{aligned}
\liminf _{n \rightarrow \infty} & \sum_{i=1}^{m}\left(\frac{k_{i}+1}{k_{i}}\right)^{k_{i}+1} \sum_{s=n+1}^{n+k_{i}} \tilde{p}_{i}(s) \\
& =\liminf _{n \rightarrow \infty} \frac{\theta}{(l-1) !} \sum_{i=1}^{m}\left(\frac{k_{i}+1}{k_{i}}\right)^{k_{i}+1} \sum_{s=n+1}^{n+k_{i}}(s-k)^{l-1} p_{i}(s)>1 .
\end{aligned}
$$

By Lemma 2.1, (2.31) has no eventually positive solution. This contradiction completes the proof.

Similarly, we have Theorem 2.7.

Theorem 2.7. Assume that $l$ is even, and the following condition holds:

$$
\limsup _{n \rightarrow \infty} \sum_{i=1}^{m} \sum_{s=n}^{n+k_{i}} s^{l-1} p_{i}(s)>(l-1) !
$$

Then every bounded solution $x_{n}$ of (1.1) oscillates.

Corollary 2.8. Assume that $l$ is even. If (2.7) or (2.26) holds, then every bounded solution of (1.1) oscillates.

In fact, (2.7) implies that (2.28) holds and (2.26) implies that (2.33) holds.

\section{Acknowledgment}

This work is partially supported by the NNSF of China (no. 10471153).

\section{References}

[1] R. P. Agarwal, Difference Equations and Inequalities. Theory, Methods, and Applications, 2nd ed., Monographs and Textbooks in Pure and Applied Mathematics, vol. 228, Marcel Dekker, New York, 2000.

[2] J. F. Cheng, Necessary and sufficient conditions for the oscillation of first-order functional difference equations, Journal of Biomathematics 18 (2003), no. 3, 295-298 (Chinese).

[3] Q. Meng and J. R. Yan, Sufficient conditions for the oscillation of non-autonomous difference equations, Acta Mathematicae Applicatae Sinica 18 (2002), no. 2, 325-332.

[4] Q. G. Tang and Y. B. Deng, Oscillation of difference equations with several delays, Journal of Hunan University 25 (1998), no. 2, 1-3 (Chinese).

[5] X. H. Tang and J. S. Yu, A further result on the oscillation of delay difference equations, Computers \& Mathematics with Applications 38 (1999), no. 11-12, 229-237.

[6] Oscillation of delay difference equation, Computers \& Mathematics with Applications 37 (1999), no. 7, 11-20.

[7] _ Oscillations of delay difference equations in a critical state, Applied Mathematics Letters 13 (2000), no. 2, 9-15.

[8] X. H. Tang and R. Y. Zhang, New oscillation criteria for delay difference equations, Computers \& Mathematics with Applications 42 (2001), no. 10-11, 1319-1330. 
[9] X. Wang, Oscillation of delay difference equations with several delays, Journal of Mathematical Analysis and Applications 286 (2003), no. 2, 664-674.

[10] Y. Zhou, Oscillation and nonoscillation for difference equations with variable delays, Applied Mathematics Letters 16 (2003), no. 7, 1083-1088.

Yinggao Zhou: School of Mathematical Science and Computing Technology,

Central South University, Changsha, Hunan 410083, China

E-mail address: ygzhou@csu.edu.cn 